GEERTZ, C. (1994). Conocimiento local, ensayos sobre la interpretación de las culturas. Barcelona: Col. Paidós Básica, Paidós.

GROSSMAN, C., MESTERMAN, S., y ADAMO, M. (1989). Violencia en la familia. Buenos Aires: Editorial Universidad.

HOOPER, C. (1994). Madres sobrevivientes al abuso sexual de sus niños. Buenos Aires: Ediciones Nueva Visión.

LARRAÍN, S., y BASCUÑ̃́N, C. (2012). 40 estudio de maltrato infantil. Santiago, Chile: UNICEF Recuperado public/pdf/maltrato_infantil.pdf

LÉVI-STRAUSS, C. (1998). Las estructuras elementales del parentesco. Barcelona: Ediciones Paidós Ibérica.

MINISTERIO DE SALUD DE CHILE Y FONDO DE NACIONES UNIDAS PARA LA INFANCIA. (2011). Guia Clinica para al Atención de Niños, Niñas y Adolescentes Menores de 15 años, Victimas de Abuso Sexual. Santiago, Chile. Recuperada el 05 de agosto de 2013 de http://www.minsal.c// portal/url/item/aaa27720f363a745e0400101le011120.pd

MOLITOR, M. (1990). La hermenéutica colectiva en Remy J. y Ruquoy. D. Méthodes dAnalyse de contenu et sociologie Louis.

PERRONE, R., y NANNINI, M. (1998). Violencia y abusos sexuales en la familia: un abordaje sistémico y comunicacional. Buenos Aires: Paidós Terapia Familiar.

PIÑA, C. (1989). Sobre la naturaleza del discurso autobiográfico. Revista Argumentos, México UAM-X, 7,131-160 SANZ, D., y MOLINA, A. (1999). Violencia y abuso en la familia. Buenos Aires: Editorial Lumen Hvmanitas.

VELÁSQUEZ, S. (2004). Violencias cotidianas, violencias de genero. Buenos Aires: Paidós

VÉLEZ, O. (2003). Reconfigurando el trabajo social: perspectivas y tendencias contemporáneas. Buenos Aires: Espacio.

\title{
Política de juventud en Chile: Cómo enfrentar la exclusión social juvenil, una mirada desde lo local
}

Youth Policy in Chile: How to confront youth social exclusion, a view from the local space

Francisca Veas Bravo

Estudiante de Ciencia Politica de la Pontificia Universidad Católica de Chile, Av.Vicuña Mackenna, 4860. E-mail: flveas@uc.cl

Daniel Castillo Mauna

Estudiante de Ciencia Politica de la Pontificia Universidad Católica de Chile, Av.Vicuña Mackenna, 4860. E-mail: daniel.castillo@ uc.cl

Resumen

A nivel latinoamericano, ha existido un auge de distintas politicas públicas de juventud, sin embargo, el problema de estas politicas radica en que plantean una visión pasiva de los jóvenes como simples beneficiarios. El presente trabajo busca aclarar esta problematica, identificando a los actores formales e informales que estan involucrados y las soluciones implementadas en torno a la política pública de juventud para el caso de Chile a nivel local, 3 temas aparecen centrales el análisis: el poder burocrático, la asesoria profesional y la canalización de las necesidades de la población

Palabras claves. Juventud, politicas públicas, política municipal, exclusión.

\section{Abstract}

There has been a rise of different public policies on youth programs at Latin American level. However the problem is that these policies have a passive view of young people as mere beneficiaries. This paper seeks to clarify this problem, identifying the involved actors and the implemented solutions for youth public policy programs for the case of Chile, at the local level, focusing specifically on the Maipú and Estación Central municipalities. In both cases an 3 themes emerged as fundamental for analysis: bureaucratic power, professional support and channeling the needs

of the population.

Key words. Youth, Public Policy, Municipal Politics, Exclusion.

\section{Introducción}

A nivel latinoamericano, las políticas públicas de juventud impulsadas por los distintos Estados "han centrado sus acciones en la educación y el tiempo libre de los jóvenes, mientras que en paralelo y en diversas etapas históricas, se han desarrollado otros modelos, centrados en el control social de sectores juveniles movilizados, en el enfrentamiento a la pobreza, desde y para la prevención del delito, y más recientemente en la inserción laboral de los jóvenes" (Rodríguez, 2000:29). El problema de estas políticas es que plantean una visión pasiva de los jóvenes como simples beneficiarios y son excesivamente asistencialistas y centralizadas, ya que la política es impulsada y ejecutada directamente desde el gobierno central.
El presente trabajo de investigación busca aclarar la problemática, los actores involucrados y las soluciones que se han dado en torno a la política pública de juventud en Chile a nivel local, y se enfoca específicamente en las comunas de Maipú y Estación Central.

En la década de los noventa se pretende dar un giro aste tipo de políticas y en ese sentido, "se ha tratado de comenzar a estructurar respuestas focalizadas en grupos juveniles concretos (asumiendo la heterogeneidad de situaciones existente), a través de estrategias que toman a los jóvenes como «actores estratégicos del desarrollo» (y no solo como beneficiarios pasivos), con enfoques descentralizados y más participativos, con la intención de promover una mayor integración social de los jóvenes a to- 
dos los niveles" (Rodríguez, 2000:29) de esta forma, el desarrollo de programas y proyectos enfocados en la juventad desde el nivel local ha adquirido relevancia y es la tendencia que deberían seguir los países que conforman América Latina. El soporte de pasta relevancia y proyec An adelante, está en que la participación juvenil se está perfilando como un catalizador de cambios, y de conscientización. De esta forma los jóvenes del pais se posicionan como objetos y sujetos de derecho.

En Chile, el área de "juventud" es una temática de largo alcance. Después del régimen militar, la generación de problemáticas económicas y políticas potenció una fuerte exclusión socioeconómica, especialmente en los jóvenes de sectores populares urbanos. Dicha problemática debió ser acogida por actores tanto estatales como de la sociedad civil englobándose en el diseño de las bases de una polí tica de juventud (Rodríguez, 2000:42).

En un inicio, la exclusión juvenil tuvo comportamientos desintegrativos importantes, creando en el Estado una visión del joven como un "problema" o una "amenaza" que debía ser enfrentada. En base a lo anterior, se creó una oferta programática que acogiera esta visión de juventud; a su vez se creó el Instituo Nacional de la Juventud, cuyo rol era solo técnico, sin lograr una representación de los jóvenes ultimo, en lo normativo estuvieron en discusión una serie de leyes de inclusión juvenil como por ejemplo la "Ley de Asociacionismo Juvenil".

Entrando al siglo XXI, a nivel local, se ha producido un cambio, ahora son las comunas quienes han considerado la necesidad de una política que acoja fomente la participación juvenil. Desde este punto de vista hubo un cambio de paradigma, ahora ya no se ve al joven como un "problema" sino que se le observa desde un enfoque de "derechos y de ciudadanía" y como "actor estratégico de desarrollo" (Programa de la Naciones Unidas para el Desarrollo, PNUD, 2009:96)

En el ámbito local, tal como señala Dávila (2005:35): "una política de juventud a nivel local, debe emanar de las instancias locales de juventud", sin embargo debe lidiar a su vez con el desarrollo de técnicas discursivas y prácticas que muchas veces se contradicen, ya que es distinto acreditar participación y organización política juvenil que plantear la idea de una documentación en la que atañen derechos y deberes, y que en este sentido, articule una relacion entre el gobierno local y lo exto quiere decir que debe exisir una correluna. Esto quiere decir que debe existir una conrelación entre la teoría y la práctica -por una parte la exis- tencia de una documentación que apoye derechos y deberes, pero por otra una institucionalidad que sea capaz de garantizar esos deberes-. De esta forma, las políticas de juventud a nivel comunal, requieren de la existencia de instancias locales de juventud, que pox en dí en Chile no se encuentan jesarollada tran desarrolladas en el nivel requerido por factores tales como "falta de madurez cívica de los jóvenes, poca experiencia acumulada en participación ciudadana, pujas por el sostenimiento de espacios de poder individuales y pujas entre la representación de los partidos políticos y los demás sectores juveniles" (Bombarolo, 2010:17)

Tal como se ha señalado, el desafío al que se enfrentan las comunas en Chile consiste en seguir la tendencia latinoamericana de desarrollo de políticas de juventud creadas y ejecutadas a nivel local, con el respaldo del gobierno central, pero esencialmente empujadas desde la propia comuna. Se presume, por lo tanto, que es más factible lograr la participación activa de los jóvenes, y focalizar la poritica, colitica, gracias al conocimiento de aquellos que forman parte de la comuna. De este modo, la satisfacción de las necesidades juveniles se constituye como un producto directamente relacionado a la importancia que se les da.

La razón de la elección de esta política pública y su problemática consiste en el abordaje y análisis de las políticas de inclusión. Este interés se enfoca particularmente en el contexto de movilización social juvenil que se ha producido en nuestro país, y en el alcance social que esta movilización han provocado, canalizando discursos de distintos sectores políticos, económicos y culturales; este fenómeno, ya sea en términos formales-institucionales, como en términos de agenda ciudadana, responde a la búsqueda de espacios de participación. Sumado a lo anterior, se desea contrastar experiencias de iniciativas comunales. Por un lado tomaremos a la comuna de Maipú, por ser un proyecto consolidado, y por otro a la comuna de Estación Central, en razón de su reciente proyecto, en formulación desde el año 2012. En la comuna de Maipú se ha dado solución a la inclusión de los jóvenes en la participación comunal por medio de un documento oficial que la misma municipalidad quiso llevar a cabo; este documento se cataliza gracias a la voluntad política existente y al esfuerzo de un grupo de administrativos de la comuna que es parte del municipio. Este proyecto se desarrolla oficialmente desde el 2011, a pesar de que sus bases se construyen desde 2004. A diferencia de Maipú, en Estación Central le requerimiento de una política de juventud se llevó a cabo a par- tir del ideal de una estudiante de Psicopedagogía, oriunda de dicha comuna, que al poseer motivaciones de realizar cambios en relación al tema juventud (como aumentar la participación ciudadana y juvenil) solicitó al programa Puentes UC ${ }^{1}$ la ayuda para la construcción de una política de juvent la constuccion de una politica de juventud, cuyo principal objetivo es realizar actividades de participación e inclusión juvenil desde la municipalidad hacia la comuna.

Estos dos ejemplos contituyen referentes para otras comunas en las que se quiera innovar en el área de juventud. Esto hace importante estudiar las d rencias que existen entre todas las comunas, tanto a nivel poblacional, como en la estructura administrativa de las municipalidades, intereses de los jóvenes, etc.

\section{Actores formales e informales}

\section{involucrados en la politica de juventud}

En la actualidad el tema de la inclusión de jóvenes al entramado nacional ha suscitado una serie de discusiones a nivel país. Sin embargo, estas no han repercutido con tanta fuerza de manera institucionalizada y formal. Por ello distintas comunas han empezado a buscar la forma de incluir a este grupo en sus programas de participación y desarrollo comunal. Una de las comunas pioneras en este ámbito fue Maipú, que desde 2004 planeaba realizar labores con jóvenes para fomentar la participación de estos en las decisiones políticas a nivel local, buscando que sus derechos fuesen más visibles y consecuentemente que su voz fuera escuchada en diferentes ámbitos. En este sentido la Dirección de Desarrollo Comunal y el Centro de Atención Familider-CAF) de la Ilustre Municipalidad de Maipú, a través de su reporte "Articulando el diseño de una política local de juventud" (2008:2), manifiestan que:

...en Chile la política de juventud posee un fuerte carácter sectorial, abordando solo temáticas vinculadas a la prevención de la violencia la drogadicción, entre otras, en tanto que las políticas de carácter universal como son las de educación, el empleo, o la salud, por nombrar algunas, carecen por completo $[\ldots]$ de estrategias de monitoreo que favorezcan la incorporación de la perspectiva de los sujetos jovenes a las cuales se dirigen, asi como tambien de anticulacion con instancias consultivas en materias de juventud de nivel central o local."

\section{Análisis de actores formale}

Para analizar el rol de los jóvenes en el desarrollo comunal se han definido ocho actores formales. Estos corresponden al Ministerio de Educación (MINEDUC), la Corporación de Fomento de la Producción (CORFO), el Consejo de la Cultura y las Artes (CCA), el Instituto Nacional de la Juventud (INJUV), las municipalidades, y los partidos políticos (pp), la Organización Internacional de la Juventud y la Comisión Económica para América Latina y el Caribe (CEPAL).

Estos actores tiene presencia institucionalizada y capacidad para general incentivos políticos y econó mico y presentan tres tipos de relación: (A) Relación de influencia directa, donde se representan vínculos de jerarquía entre instituciones y donde se reflejan instancias de superioridad de recursos entre las dos instituciones; (B) relación de trabajo conjunto donde se reflejan instancias en que hay posibilidades de llevar a cabo proyectos con recursos mixtos (gubernamental-municipal); y (C) relación irregular-temporal, donde se reflejan relaciones de apoyo político temporal a ciertos proyectos e instituciones en espacios de tiempo específicos. Estas relaciones se pueden observar en la Figura 1.

Estas redes han sido clasificadas de acuerdo a su desempeño en siete categorías, de acuerdo a lo señalado por el reporte "Historia, Procesos y Desafíos de la institucionalidad de Juventud: El caso de la Oficina de la Juventud de Maipú", del año 2011, de la Ilustre Municipalidad de Maipú. En este reporte, las áreas de acción juvenil corresponden a (i) la participación y la representación, (ii) la educación (iii) emprendimiento y la empleabilidad, (iv) la salud, (v) la cultura y la recreación, (vi) la familia, y (vii) el acceso y uso de tecnología. Cabe señalar que no todas las categorías han sido consideradas, esto guarda relación con la extensión y con la pertinencia del trabajo, donde no siempre aplica la categoría al actor señalado

A pesar de que el diagrama puede dar cuenta de las relaciones y las posibilidades de agencia entre los distintos actores, es necesario establecer qué es lo que hace cada uno, y cómo se enfocan en cada una de las áreas propuestas. En esta línea, se detallan generalizadamente los siete actores formales que hemos estimado pertinentes en torno a la política de juventud.

\footnotetext{
Puentes UC es un programa del Centro de Politicas Públicas UC cuyo fin es desarrollar iniciativas orientadas a la solución de problemas
reales a nivel local. (puentesuc.cl).
} 
FIGURA No1

RELACIONES DE ACTORES FORMALES

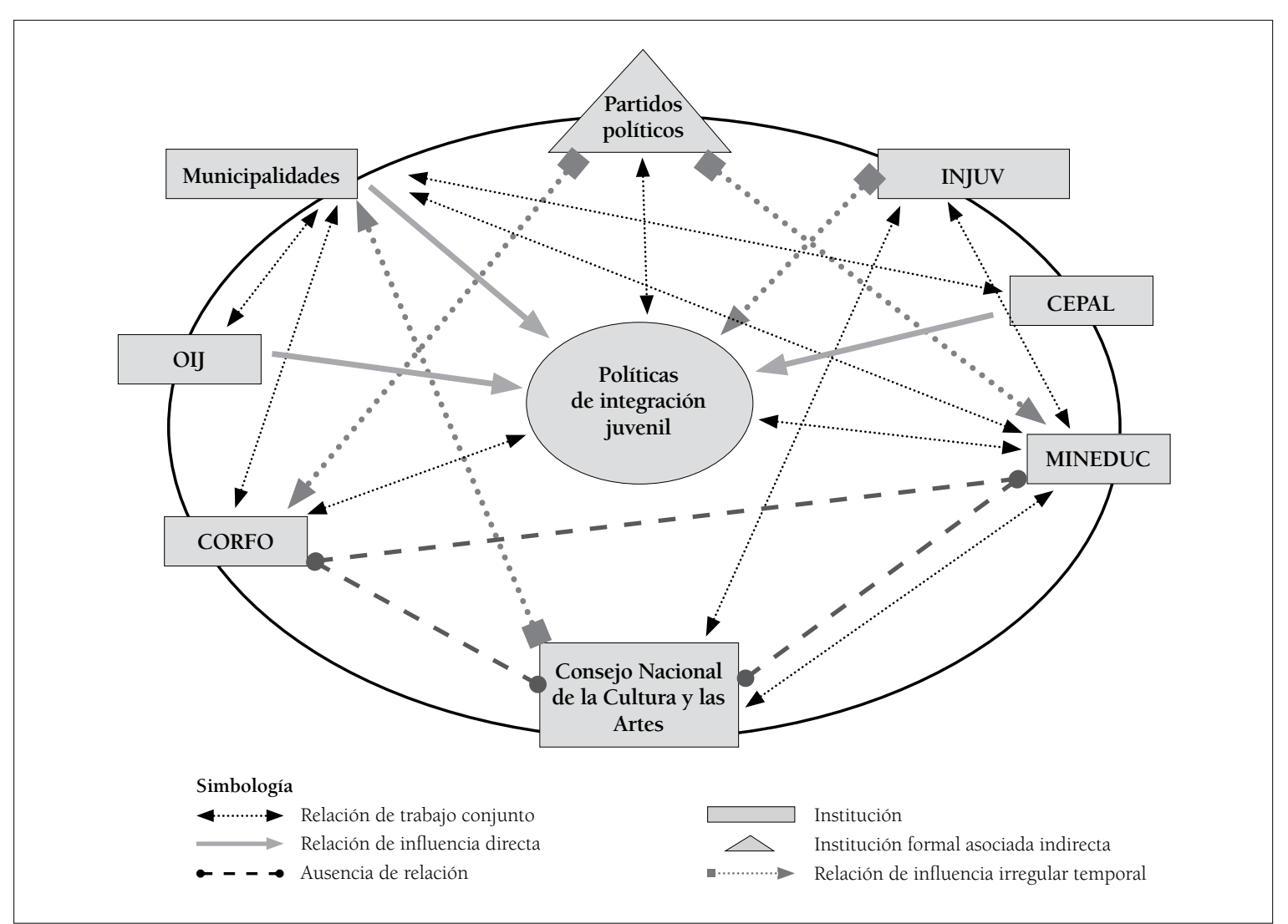

Fuente: Elaboración propia (2012)

a. Municipalidades: Son las instituciones formales que quizás han tenido la mayor influencia en el desarrollo de políticas sectoriales de juventud. A través de los departamentos de desarrollo comunitario han logrado establecer no solo planes y programas orientados a satisfacer las necesidades de los jóvenes, en for rantías mínimas sino que también han tendido a establecer instancias regulares de catastro y consulta sobre necesidades comunales de la juventud. En el cas de la Municipalidad de Maipú, se han llevado a cabo reuniones plenarias y asambleas comunales de juventud con el fin de reunir inquietudes y plantear proyecciones sobre las condiciones sociales-politic sociales-polic ciativas se establ instancias de representación y participación efectiva en política local.

Con posterioridad a estas reuniones plenarias, que según reportes de la municipalidad datan del año 2008, se han llevado a cabo paneles expertos sobre temáticas de educación, emprendimiento y salud, con el fin de implementar políticas sec- toriales en base a las propuestas programáticas derivadas de los ministerios asociados; a saber, nil, en torno al área de emprendimiento, se han enfocado bajo el alero de los planes de "capital para la contracion d CORFO, y han buscado para la contratacion de $C$ de planes gubernamentales. En este contexto, el de asesor experto) han llevado a cabo planes complementarios de políticas nacionales de inclusión, como la prevención de drogas y alcohol y el aumento de las tasas de escolaridad básica y media.

Respecto a temas de cultura y familia, se ha optado por el fomento municipal de ferias del adulto mayor, ferias vocacionales, ciclos de cine, teatro y danza, y jornadas deportivas, que han buscado sentar precedentes en cuanto a inclusión familiar y el potenciamiento de bases de integración para la comunidad. Si bien no se distingue una mayor preferencia por el sector juvenil dentro de la plala implementación de políticas de inclusión juvesemilla", microemprendimientos, y subsidios posicionar la municipalidad como foco de acción MINEDUC y el Ministerio de Salud (en calidad nificación de los proyectos, estos se encuentran como parte del público objetivo.

b. Instituto Nacional de la Juventud: Si bien el INJUV puede ser entendido como uno de los actores más pertinentes, en el caso de la política de juventud de Maipú ha tenido un rol acotado, orientándose al fomento de políticas de participación y ciudadanía, educación y emprendimiento, empleabilidad, recreación y desarrollo familiar y salud. En cuanto a políticas de participación y ciuct esempeñado labores de coordinación con agencias gubernamentales para asesorar temas como volatilidad electoral y participación política juvenil; en temas de educación, emprendimiento y empleo, y salud. Ha actuado también como agente coordinador al entregar información estadística sobre sectores vulnerables y necesidades básicas, así como información sobre la calidal de los servicios que lormación sob existen actualmente para la juventud. También ha tenido un rol significativo al definir a la juventud como agente económico importante y ha orientado campañas sobre descuentos con la "tarjeta joven". En cuanto a temas de desarrollo familiar, cultura y recreación, el INJUV ha tenido un amplio rol como "agente precursor" al diseñar programas de otras instituciones asociadas como la Defensoría Penal Púbica, el Servicio Nacional de Menores (SENAME) o el Servicio Nacional de la Mujer (SERNAM) mediante charlas sobre desarrollo familiar, prevención de violencia doméstica y orientación para madres y padres solteros.

c. Ministerio de Educación: El MINEDUC ha contribuido al desarrollo de temas como emprendimiento, salud, cultura y recreación

Respecto al emprendimiento y empleabilidad, el ministerio ha desarrollado planes de fomento a la educación técnica con el fin de apoyar a los sectores vulnerables que no pueden optar a la educación superior y cuya "herramienta de salida" es la educación técnico-profesional. Para esto, ha logrado establecer un marco referencial sobre carreras técnicas con demanda laboral, que permite asegurar cupos de trabajo, y ha llevado a cabo campar para promover la iniciativa a esudir como motor socil. En terinos de salud, el MINEDUC ha llevado a cabo planes de asociación con el Ministerio de Salud y el Servicio Nacional para la Prevención y Rehabilitación del Consumo de Drogas y Alcohol (SENDA) para promover el auto-cuidado escolar en consumo de drogas, alcohol y también ha desarrollado planes sobre educación sexual temprana en colegios con amplias tasas de embarazo juvenil.

En relación a temas de cultura y recreación, y familia, el MINEDUC ha llevado a cabo proyecto sobre fomento de actividad deportiva familiar, la ampliación de muestras culturales (como invitaciones a museos, charlas, talleres, etc.).

d. Consejo de la Cultura y las Artes (CCA): El CCA ha actuado como órgano asociado más que agente precursor, buscando acercar actores institucionales respecto a las políticas públicas de cultura. En este contexto, ha llevado a cabo reuniones multinivel donde ha presentado organizaciones ante la mivicipla para lograr un acuerdo base sobre difusión y promoción de eventos culturales. En razón de su rol asociado, el CCA ha tenido una participación limitada a la difusión de panoramas culturales y la apertura de espacios a grupos o asociaciones nuevas.

e) Corporación de Fomento de la Producción (CORFO): Esta institución se define como acto de importancia en razón de su amplia capacidad para movilizar recursos. En este sentido su aporte a la participación juvenil ha consistido en el ofrecimiento de oportunidades y de proyección. En términos de Educación, ha permitido el desarrollo por buscar instancis de perfeccionamiento profesional para poder optar a capitales y fondos de inversión, por lo que su aporte educacional se enfoca a la promoción de incentivos en proporción a la capacidad profesional (que dentro de los canales formales se simboliza con la educación y/o el perfeccionamiento).

En relación a su aporte al emprendimiento y la empleabilidad, CORFO ha cooperado con las municipalidades en promover puestos de trabajo para que la juventud postule y participe, también ha abierto espacios de educación, capacitación/ certificación para jóvenes que deseen aprender o especializarse en un oficio, puedan hacerlo de manera asequible. Otra perspectiva del aporte de CORFO para el emprendimiento consiste en la apertura de fondos concursables para ideas innovadoras y que permitan la generación de flujos de capital autogestionado.

En relación a salud, recreación y cultura, CORFO ha tenido una participación marginal por centrarse en el desarrollo económico de las política de juventud. Ahora, en relación a uso y pro- 
moción de las tecnologías, la Corporación ha desarrollado una amplia plataforma de difusión información y capacitación

f. Partidos políticos: El aporte principal de los partidos políticos se desarrolla en torno a la apertura de espacios de participación de la juventud, el fomento de la educación, la búsqueda del emprendimiento, y la revalorización de las "familias". Los espacios de participación que los partidos políticos han abierto se relacionan con partidos policos han abieto se relacionan con la formacion de cuerpos politicos juveniles $y$ la ormación de cultura civica mediante la promoción del voto y la participación electoral. En este sentido, la habilidad de los partidos políticos se concentra en el levantamiento de figuras públicas que relacionen política con juventud en torno un discurso de cambio y renovación"

g. Dos organismos internacionales se reconocen como relevantes en la inclusión juvenil la Comisión Económica para América Latina y el Caribe (CEPAL) y la organización Iberoamericana de la Juventud (OIJ). La CEPAL ha contribuido con información para caracterizar a la población juvenil La OIJ con ribuýó originalmente con esjuveni. La OIj contribuyó originalmente con estudios que favorecieron la mejor implementación
de programas juneniles

FIGURA N 2

ACTORES INFORMALES

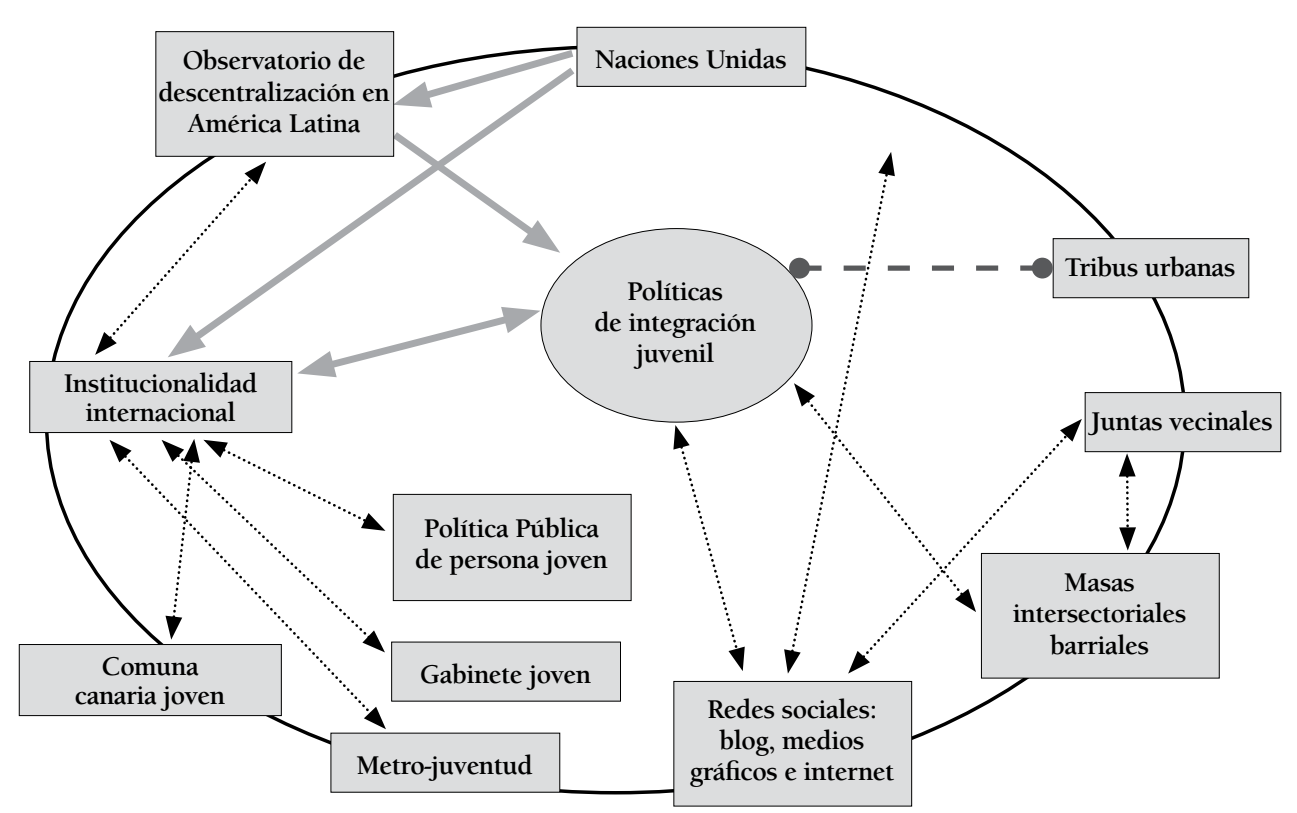

Simbologia

$\rightarrow$ Relación de trabajo conjunto

$\longrightarrow$ Relación de influencia directa

$\ldots$ Ausencia de relacio

$\square$ Institucio

\section{Análisis de actores informales}

Para este análisis se propone dar cabida a la cantidad de actores y redes que influyen de manera indirecta en la formulación y estabilidad del fin emprender. Es decir, no son influyentes de form a directa e institucionalmente no se presentan como organismos o actores con "mayor poder" dentro del entramado de actores, pero sí en algunos momentos de la formulación de la política se presentan como thentes, negativa o positivamente, en ella.

En este caso particular, y como se observa en la Figura 2, resulta difícil clasificar a las organizaciones o actores en grupos, debido a que corresponden a redes que no se identifican con una institucionalidad oficial, por lo que en este mapa no se ha considerado esa posibilidad. Luego se observa que los actores parecieran tener una relación casi unidireccional con la política en sí, debido a que su actuar en un principio fue el de proveer conocimiento a la formulación de la política contextualizando o entregando una base informacional a su creación. Consiguientemente la actuación de estas redes cambiaron mientras la política se implementaba, demostrando la esencia de la formulación de estos mapas de actores, el hecho de que con el pasar del tiempo, éstos puedan variar.

uente: Elaboración propia (2012).
En un principio, las relaciones entre política de juventud y actores era más bien unidireccional, así se observa con las distintas tribus urbanas de jóvenes, mesas intersectoriales barriales, juntas vecinales fueron reunidas para vecinales, ellas fueron

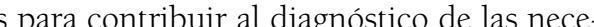
sidades juveniles a nivel comunal. Posteriormente, la relación únicamente informativa se transformó en una relación de retroalimentación con las organizaciones de base ya mencionadas

Las organizaciones más institucionalizadas como juntas de vecinos y mesas intersectoriales tuvieron as como las tribus urbanas que son menos permanentes en el tiempo.

En la difusión de estos programas y proyectos, es muy importante la actuación de las redes sociales, donde las personas y actores involucrados pueden contactarse para realizar actividades por medio de blogs, internet y medios gráficos. Así, el hecho de que la política haga uso de este medio es fundamental habiendo una relación unidireccional desde los programas y proyectos hacia las redes sociales, y de estas al resto de las organizaciones tanto formales como informales.

Dentro de los actores informales que influyen indirectamente en la formulación de los proyectos de integración juvenil están los actores no gubernamentales e internacionales que interfirieron en que la política. Así, existe el Observatorio de la Descentralización en América Latina de FLACMA (Federación Latinoamericana de Ciudades Municipios y Asociaciones de Gobiernos Locales), que gracias a sus estudios pudo nutrir el contenido y resultado de la formulación de proyectos que se estaban proponiendo para la política. La FLACMA es una organización propositiva de libre adhesión que representa a los 16.132 municipios, intendencias y prefecturas, a las ciudades y a las asociaciones de gobiernos locales, redes y gremios, municipalidades de América Latina y El Caribe (FLACMA, s.f:s.p). Gracias al apoyo de esta organización es posible la evaluación y establecimiento de políticas sociales en el medio local, aunque pocas municipalidades de Chile tengan contacto o hayan recurrido lidades de Chile tengan contacto o hayan recurrido la 'Política Pública de la Persona Joven' en Costa Rica, 'Comuna Canaria Joven' en Uruguay, 'Metrojuventud' en Colombiam Gabinete Joven en Argentina, que han servido como base e influencia natural para que los programas juveniles en Chile consideren la inclusión de los jóvenes en etapas de formulación de programas y política en el marco de relaciones bidireccionales entre distintos actores involucrados

\section{Análisis de interfaz para el}

\section{entendimiento de una propuesta de} intervención

Un enfoque que complementa el concepto de exclusión social es el enfoque de interfaz, acuñado por Norman Long. De acuerdo a este enfoque, en el nivel microlocal y regional interactúan una serie de actores, individuales o colectivos que representan diferentes cosmovisiones y miradas de mundo: "La noción de interfaz social se torna relevante como una forma de explorar y entender problemáticas de heterogeneidad social, diversidad cultural y los conflictos inherentes a los procesos que involucran la intervención externa" (Long, 2001:65).

Bryan Roberts identifica seis posibles interacciones entre actores en la interfaz, siendo una de ellas el "excluyente". "La exclusión como es clasicamente definida por Simmel en 1908 se refiere a una relación de ciudadanía de segunda clase en que los jóvenes son miembros de la sociedad, pero la ayuda que reciben del Estado no es otorgada para asegurar su igualdad como ciudadanos, sino para mantener el tejido social" (Roberts, 2001:14)

Además, enfoca su análisis en el rol del gobierno local en la política social y descentralización para acabar con la exclusión. Bajo esta perpectiva podemos analizar la política de juventud que ha privilegiado una orientación hacia la participación constante desde lo local hacia lo comunal. Por un lado, en Maipú se tiene algo mucho más estable y estructurado, pero po otro lado, en Estación Central no se presenta una mayor planificación al respecto.

Ante esta situación, se ha planteado como referencia leórica a las percepciones de comunidad y su vínculo con la participación ciudadana con el objetivo de aproximar la perspectiva de interfaz para el análisis de la política de juventud.

\section{Nociones de comunidad y participación}

\section{ciudadana}

Dentro del estudio de la democracia y la participación ciudadana es posible encontrar cierto debate sobre cuáles son los mecanismos que permiten un empoderamiento real de la ciudadanía. En este sentido, las percepciones de comunidad se definen como una primera aproximación a los estudios sobre democracia porque definen las esferas de participación que los sistemas políticos pueden tener, y también los alcances que la dignidad humana tiene sobre la estructura socio-política. 
En esta línea, las nociones de comunidad que involucran componentes de identidad, reciprocidad, y participación, evolucionan en mecanismos de deliberación política en razón de los tipos y magnitudes de incentivos que son capaces de movilizar, as posibilidades de apoyo y respaldo ciudadano, $y$ las capacidades de lograr efectos deseados; es decir, finalmente, la definición de comunidad permite distintas formas de participación democrática en cuanto da cabida a las manifestaciones individuales y equilibradas de sus participantes.

Sin embargo, no hay una relación natural entre los componentes de identidad y la democracia. Esto se debe a que a pesar de que la comunidad tenga un sentido de identificación como grupo, los grados de institucionalización de los vínculos son los que otorgan validez a las redes informales a la hora de lograr acuerdos o la obtención de resultados. Por lo que la formación de obligaciones -o acuerdos vinculantes- pueden crear bases estables de convivencia en comunidad Así, la formación de un marco de gobernabilidad comparida y de lógica de poder colegiado se logra a través de la definición de patrones de reconocimiento y legitimación mutua que permitan a los actores de una comunidad sentirse parte íntegra de un grupo social.

En este trabajo en particular, la interfaz ha incluido 2 casos de estudio la comuna de Maipú y la comuna

de Estacion radamente por comuna, para proponer una entreg

\section{Maipú: una política de juventud ya}

\section{establecida}

En el caso de la política de juventud de Maipú, la percepción de comunidad juvenil, hasta el año 2008, se encontraba alejada de las posibilidades reales de participación social, y solo se reducía a la formación de grupos de interés particular -grupos de baile, clubes deportivos, etc.-. Sin embargo, en el año 2008, el alcalde de la comuna, en conjunto con la Dirección de Desarrollo Comunal, decide poner en marcha la planificación y articulación de una política comunal de juventud que pudiera ser el reflejo de las posibilidades de participación de los jóvenes en la vida comunitaria. Esta iniciativa contó con diversas asesorías profesionales, donde se destaca el rol del Fondo de las Naciones Unidas para la Infancia (UNICEF) y de la Defensoría Ciudadana, que permitieron el desarrollo de un reglamento municipal que otorgara la calidad de "normativas vinculantes" a las iniciativas de promoción comunal, incluyendo a la política de juventud, como parte de las obligaciones jurídicas del municipio hacia sus habitantes.

De acuerdo a esto, el establecimiento de un programa con obligación jurídica permite asegurar el "derecho a participar y a identificarse" con la comuna a través de la creación un marco regulatorio quna a través de la creación un marco regulatorio que reconozca y habilite la comuras sociales y gubernamentales en razón de la definición de las personas como agentes de cambio y no solo como meros habitantes.

Respecto a lo anterior, la oficina Maipú Joven -encargada de la planificación y desarrollo de la política de juventud de Maipú- tiene por objetivo el desarrollo de una estructura normativa y funcional que refleje la intención y obligación del municipio de desarrollar estrategias de vínculo social. En esta línea, Ignacio Corcuera, encargado de la oficina Maipú Joven, manifiesta que las posibilidades que otorga el desarrollo de una política de juventud van más allá del número de personas que son escuchadas, sino que se orienta a la posibilidad de entregar el derecho a participar y de influir políticamente a actores que muchas veces no son tomados en cuenta como agentes de toma de decisión. Esto, en relación a la deliberación y la comunidad, permite constatar que los grados de identificación como grupo cuentan con un respaldo administrativo que (1) los reconoce como parte de su población infu(1) los reconoce como par calidad de promotor y facilitador de instancias. Sin embargo, y a pesar del soporte administrativo con el que este organismo cuenta por parte de la municipalidad, las iniciativas de este tipo presentan dos problemas importantes. El primero se relaciona con la cantidad de personas a las que se incluye dentro del programa, y el segundo se relaciona con los tiempos de trabajo y reacción entre los agentes involucrados.

Respecto al primero, la política de juventud de Maipú tiene dificultad para llegar a toda la comunidad juvenil por las dimensiones de la misma comuna. En este sentido, la estrategia que ha utilizado Maipú Joven como el municipio ha sido incluir representantes de las diversas asociaciones juveniles y solicitar apoyo de los mismos asistentes para llevar a otros jóvenes a las reuniones, generando así un "efecto bola de nieve" entre los participantes.

El segundo problema es quizás uno de los más usuales en la definición de políticas públicas, y guarda relación con las posibilidades y alcances que los jóvenes esperan del municipio, y las posibilidades que tiene este para llevar a cabo los proyectos.
En referencia a este problema, la solución que se ha aplicado es la de utilizar mecanismos de relación informal entre Maipú Joven y el Municipio, con el fin de acelerar procesos a través de la cooptación de instancias de poder superior al organismo que . Joven ha debido valerse de la relación personal entre su encargado y el Alcalde con el propósito de que él mismo sea quién otorgue rapidez al proceso. Si bien este tipo de iniciativas se encuentra dentro de la lógica organizacional esperable del poder subnacional, no corresponde al ideal en cuanto a la valoración que ciertos organismos otorgan a los proyectos de juventud. En este sentido, la a proyectos de blematica subyacente se define en torno a ciertos departamentos que traban los proyectos -por diversos motivos- haciendo que los tiempos esperados y los posibles sean discordantes. Obligando a Maipú Joven a recurrir a vínculos extra-institucionales para devolver o acelerar los procesos.

Ahora, dentro de la temática de "comunidad juvenil" hay un punto importante, y se enfoca en el valor que se otorga a la legitimación por parte de los jóvenes sobre la política. En referencia a esto, Corcuera manifiesta que en una primera instancia los jóvenes invitados a la primera sesión de reunión se mostraron desconfiados respecto del rol que podía jugar la Municipalidad en su favor. Sin embargo, con el paso del tiempo, estos fueron disipando su desconfianza y se mostraron abiertos a recibir y apoyar al municipio en su labor de promoción, definiéndose ellos mismos como agentes de cambio al contar con la legitimación de la Municipalidad.

Otro punto importante de señalar en el estudio de la comuna, se enfoca en el valor que se le otorga al poder colegiado como mecanismo válido dentro del desarrollo de políticas subnacionales. En esta línea, se han identificado dos frentes, el primero se relaciona con el poder colegiado en la etapa formativa de la norma, y el segundo se enfoca en la atomización de poder en la implementación de la política. En el caso de la formación, el poder colegiado se observa en dos casos: El primero se enfoca en el "levantamiento de la iniciativa" por parte del alcalde hacia otras esferas profesionales, con el fin de desarrollar un proceso más transparente. Esto se llevó a cabo a través de la realización de asambleas ciudadanas y consultas comunales con el fin de constatar cuáles eran las necesidades y objetivos a satisfacer con una política de desarrollo social comunal.

El segundo caso se observa en la voluntad de desarrollar una normativa en conjunto a otros actores expertos y especialistas. La posibilidad de contar con el apoyo de la UNICEF no es menor en razón de que valida el procedimiento y las intenciones a nivel internacional, y la posibilidad de contar con la asesoría de la Defensoría Ciudadana marca un punto importante a la hora de considerar seriamente el tema de la jurisprudencia de la política.

Respecto de la implementación es posible observar instancias democráticas-deliberativas en plenitud. El desarrollo de las asambleas con los jóvenes, que se realizan el primer sábado de cada mes, es una señal relevante en cuanto refleja la periodicidad de os vínculos entre los organismos municipales y la sociedad. También, los vínculos de confianza, en elación a lo manifestado por Ignacio Corcuera, son una señal importante, pues representan mejor la pertinencia y desarrollo de la política. Esto se debe que son los mismos jóvenes los que tienen la posibilidad de mejorar y proponer nuevas formas de desarrollar la política juvenil y de enfocar de mejor manera los proyectos que propone el municipio. En relación a lo manifestado por Corcuera, las posibilidades de contar con retroalimentación directa son escasas, y deben ser tomadas en cuenta porque son formas rápidas y constructivas de hacer política.

Ahora, hay preguntas importantes que se desprenden de la política de juventud de Maipú: ¿Por que se considera a los jóvenes?, ¿Cómo se trabaja con los jóvenes?, ¿Qué hace el Estado para subsanar estas necesidades en Maipú?, y ¿Qué lecciones se desprenden de la política de juventud de Maipú? Los jóvenes, en relación a lo manifestado por Ignacio Corcuera, han sido tomados en cuenta porque actualmente no hay instancias donde la juventud chilena pueda sentirse parte de la estructura política. Son parte de un juego de utilización y de exclusión de participación a la hora de tomar decisiones respecto a su propio contexto; privándolos así, de poder ayudar a crear mejores políticas públicas y tomar en serio su rol de ciudadanos.

Los mecanismos de trabajo que se han utilizado en esta comuna se relacionan a la programación de reuniones -a través del denominado Senado Juvenil- que se realizan una vez al mes, y donde se recoge periódicamente la opinión de estos jóvenes respecto de sus necesidades; y también se ha considerado el rol de las autoridades y organismos expertos que puedan proveer de asistencia técnica -tanto en el proceso de formación de la política como en el desartollo de estas- Estas dos formas en elítica sea más precisa y directa en l sentido de que hay una relación más espontánea de retroalimentación, donde "hay que tratar de res- 
ponder de la mejor forma posible a las inquietudes y requerimientos que ellos presentan

Las necesidades, de acuerdo a lo planteado por Corcuera se orientan más hacia un sentido positivo más que punitive una política de juventud que destaque las potencialidades de los jóvenes respecto del valor de su participación y cooperación en la política comunal más que el desarrollo de estrategias asistencialistas. Respecto a esto, Ignacio Corcuera manifiesta que las instancias de solidaridad, como las colonias juveniles y el fomento del uso de espacios públicos ha sido positivo para reafirmar la pertenencia de los jóvenes en la comunidad maipucina.

Respecto del rol del Estado, es posible observar constatar que no hay un rol preponderante de este en Maipú. Pues este no ha otorgado mayores recursos para el fomento de la política de juventud, delegando esta tarea de cohesión social a los mumicipu unicamente, instancias donde se ha visto cooperación se ha dado por el rol marginal del Instituto Nacional de Juventud, a través de la implementación de salas de computación para la comunidad.

Finalmente, las lecciones que se han podido constatar respecto de la idea de comunidad juvenil en "identidad juvenil" a través del diálogo, la inclusión, la discusión y la proposición que los jóvenes de la comuna pueden hacer por su propio bienestar y desarrollo, y por el rol constructivo respecto de su propia imagen dentro de la sociedad.

\section{Estación Central: los inicios de una}

\section{formulacion de politica de juventud}

En la comuna de Estación Central el caso es muy distinto. Daniela Flores es estudiante de Psicopedagogía y desde el agosto de 2012 está trabajando diariamente en la municipalidad como encargada del departamento de juventud.

Esta área de trabajo está recientemente creada, siendo Daniela la única participante. Por lo anterior, buscando ayuda en la formulación de bases legales de una política de juventud recurrió al programa Puentes UC para solicitar ayuda a un grupo de estudiantes, de esta forma tendría un documento que pudiese habilitar su actuación dentro de la municipalidad para luego proponer un presupuesto y consecuentemente se institucionalizara esta área dentro de la municipalidad.

La intención de participar en juventud nació de un grupo de Encuentro de Jóvenes en el Espíritu (EJE) de la misma comuna, quienes al percatarse de que la municipalidad hacía poco por juventud y sintiendo que los servicios eran entregados de forma disgregada para los jóvenes, decidieron plantear esta inquietud ante el municipio. A partir de lo anterior el mismo edil y consecuentemente el director terior, el mismo edil y consurection de Desarollo Comun (DIDECO) (DIDECO), le propusieron a Daniela hacerse cargo de este departamento asumiendo el rol de líder para poder avanzar en esta temática. Sin embargo, pronto se percató de que sin tener las bases correspondientes para la acción no podía hacer muchos cambios dentro de esta área. Más adelante y con la ayuda de Puentes UC ha logrado crear un marco o bases para una política de juventud, teniendo como referente la política de juventud creada y aplicada en Maipú. Sus dificultades han sido más institucionales, por ejemplo, (1) la municipalidad no posee programas que solamente se focalicen en la población juvenil debido al desconocimiento sobre esta población, (2) todo el trabajo de juventud es actualmente atendido por una sola persona y solo con apoyo del director de la DIDECO y ahora del encargado de Cultura de Estación Central, y por último (3) la asignación de recursos económicos para poder crear una estabilidad dentro de la oficina resulta una problemática de suma urgencia para resolver, ya que entonces este deja de ser un departamento activo sin el capital social para fomentar el trabajo en juventud.

Sin embargo, es dificultoso cambiar la visión "paradigmática" que se tiene de la juventud dentro del municipio, en que se observa al joven desde un prisma de problema y no como un actor generador de cambios, además. Desde la misma institución se tiene la percepción de que los jóvenes no tienen inquietudes políticas ni sociales. Para Daniela ha sido un error pensar de esta manera. Haciendo intentos de cambio en esta visión, decidió hace un tiempo crear lazos con organizaciones juveniles de la comuna percatándose de que otro gran problema es el hecho de que entre las organizaciones juveniles no existe un contacto continuo, ni ayuda mutua y tampoco existe un canal de comunicación efectiva. Todo lo anterior ha ayudado a comprender que es necesario primero sentar bases municipales de participación para luego fomentar la participación entre organizaciones con razones mucho más establecidas.

La importancia que Daniela le da a este tipo de herramientas es la ayuda que habría hacia los jóvenes no solo enfocada en los estudios y trabajo, sino que abarcando una serie de temáticas más agudas y que en ello existiese información detallada del por qué enfocarse en un tema y no en otro. Para ello conocer más a los jóvenes de su comuna es fundamental. Por otro lado, durante la entrevista hace un énfasis implícito en que lo local en esta temática es mucho más relevante que lo que se puede hacer a nivel nacional, ya que los conocimientos sobre la comuna pue la colaboración por parte de instituciones como el Instituto Nacional de la Juventud (INJUV) es poco focalizada y no entiende la esencia de los jóvenes de cierto sector, ni comprende qué es lo que realmente necesitan.

Hasta el cierre de este artículo el trabajo en conjunto con Puentes UC aún no había finalizado por lo que el impacto de esta polica en construcc lo que no era observable aún. Tampoco se pudía observar una implementación pero sí los intentos de la encargada por dar a conocer a distintas organizaciones juveniles y jóvenes en general de que estaba en construcción una herramienta desde la municipalidad para fomentar sus derechos y deberes. Lo anterior, en un inicio, no tuvo una gran acogida, pero muchos han decidido participar de las distintas reuniones que ella ha programado en la municipalidad comprendiendo luego que esto es para ellos y por ellos, sintiendo el empoderamiento para poder decidir qué es lo que muetra que el diseño de la política ha incluido a los jóvenes

Aún se espera el diseño final de la política para presentarla ante el consejo municipal y ver sara aprobada o no para su implementación, y formalizar lo que será el departamento de juventud en Estación Central.

\section{Plan de accion para una intervencion:}

\section{los lineamient}

juventud efectiva

De acuerdo a lo discutido podemos señalar que para la existencia de una política juvenil efectiva es de suma importancia que existan los siguientes puntos:

- Voluntad política local.

- Reconocimiento de la población joven como un actor generador de cambios positivos de su realidad y actor de derechos.

- Capacidad técnica que permita enfrentar dificultades relacionadas con la municipalidad y, a su vez, con este ámbito.

- Posibilidad de influir, por parte del equipo que conforma el departamento de juventud, en decisiones dirigidas a actividades juveniles, incluida la capacidad de decidir si se aplica o no un programa proveniente del gobierno central.

- La existencia de una amplia programación muni- cipal en torno al ámbito juvenil

Además es que exista participación, difusión presupuesto.

\section{Participación}

La participación ciudadana debiese ser entendida como "un proceso abierto, transformativo, en el que se lleva a cabo una política específica orientada capacitar y aumentar el poder de la ciudadanía, y a impulsar su papel en el fortalecimiento del desarrolo comunitario" (Camps, 2000:233).

Para llevar a cabo esto es necesario que exista participación en distintos niveles como proporcionar información, trabajo compartido, decisión conjunt y empoderamiento (Camps, 2000:234). Esto result fundamental, y así fuese observado en las experiencias comunales estudiadas, por ejemplo en Estación Central es una constante el hecho de reunir a los actores fundmentales para recoger información y a su vez generar empoderamiento de los jóvenes hacia esta nueva herramienta a utilizar.

\section{Difusión}

a difusión es relevante en cuanto no se desliga de lo que es la participación. Si bien en Maipú se observó un deficit en esta area, han logrado convocar y conformar un Senado Joven que colabora en la toma de decisiones que el municipio debe hacer en torno a la temática de juventud. Esto es importante, debido a que existe en ellos un tipo de difusión que llega a ser "bola de nieve" al intentar fomentar la participación entre amigos, familiares, etc. En cambio, en Estación Central, es posible ver que el hecho de reunir a organizaciones para recopilar información fomenta la participación de los jóvenes pertenecientes a esas organizaciones los que se motivan a participar de la construcción de la política.

Esto último, a su vez, es una problemática a resolver porque la difusión sobre el accionar de los departamentos es importante para motivar a los ciudadanos a participar.

\section{Presupuesto}

Es necesario un presupuesto fijo y claro para poder crear un ordenamiento y estabilidad dentro del deente la política de juventud. Es probable que el tipo de presupuesto que sea necesario crear sea el de uno participativo, en que "los habitantes recib[en] ... información sobre el presupuesto y su ejecución, e identific[an] sus propia prioridades $[\ldots]$ permitiendo el acceso masivo de sus habitantes a la toma de decisiones presupuestarias [...]" (Camps, 2000:247). 
Esto resultaría beneficioso porque, según Camps, (2000:248) permite:

- Comprender problemas cruciales en torno al área de juventud.

- Establecer prioridades de los problemas que requieren de una inmediata atención.

- Seleccionar prioridades y generar soluciones prácticas.

- Decidir sobre programas concretos y planes de inversión.

- Evaluar y revisar éxitos y fracasos del programa para mejorar sus criterios.

\section{Proyecciones, aprendizajes y}

\section{recomendaciones}

Después de lo anteriormente expuesto, y considerando la situación de Maipú y Estación Central, es posible establecer que las dinámicas de integración y de deliberación a través del desarrollo de estrategias de inclusión juvenil tienen que gozar de una base estable de compromiso social y de convicción política para ser implementadas exitosamente. En este sentido, la comuna de Maipú es un buen referente sobre cómo llevar a cabo un plan y estrategia de integración social por cuanto permite la interacción de múltiples actores y la negociación de intereses difusos. Estas lecciones, en referencia a las intenciones que se han manifestado en Estación Central, deben ser aprovechadas para igualar y mejorar las políticas de desarrollo comunitario orientando las condiciones existentes y no solo a través de la creación de nuevos mecanismos.

Respecto de las proyecciones y recomendaciones, las estrategias de difusión y de canalización de inpara llevar a buen puerto las estrategias comunales, porque permiten dotar a las iniciativas y autoridades de la legitimación necesaria y del componente de reconocimiento que la definición de comunidad sugiere. En este sentido, la difusión no debe ser tomada como un proceso inicial, sino que debe ser una parte integral del proceso de construcción debe ser consicter

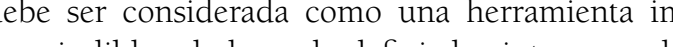
prescindible a la hora de definir los intereses y las proyecciones de las políticas, porque es este componente el que permite la retroalimentación necesaria para ir adaptando los contextos e ir adecuando los procedimientos.

Finalmente, los aprendizajes derivados del estudio de la política de Maipú permiten señalar tres cosas importantes. La primera se refiere al poder burocrático que sustenta los proyectos sociales, el que debe ser reconocido y respetado con el fin de evitar desvíos e irregularidades. El segundo punto se relaciona con la necesidad de contar con asesoría profesionat; este componente es importante no solo en la etapa de formación de la política, sino que también a la hora de implementarla, pues va ajustando los presupuestos, las necesidades y los alcances de los proyectos. Y el tercer componente a rescatar tiene relación con las necesidades que presenta la población, donde se debe enfocar un proyecto que sea acorde a las dinámicas sociales que se presentan y no solo corresponder a la implementación ciega de programas provenientes de otros sistemas.

En síntesis, las dinámicas de construcción de capital social van mucho más allá de lo visible y lo aparente, también se debe considerar el tiempo y el espacio donde están insertas las personas y donde nacen las necesidades, porque son finalmente estas las que delimitan las voluntades políticas, los recursos y el capital humano que decide poner en práctica una política de desarrollo comunitario que tenga el respeto por la identidad como un factor fundante.

\section{Referencias}

BIBLIOTECA DEL CONGRESO NACIONAL DE CHILE. (2012). Reportes Estadísticos Comunales 2012: Estación Central. Recuperado el 24 de octubre de 2012: http://reportescomunales.bcn.cl/2012/index.php/ Estaci\%C3\%B3n_Central

BOMBAROLO, F. (2010). Informe final: "Hacia la definición de una política de juventud para la comuna de Maipü. Santiago: Organización Iberoamericana de Juventud.

CAMPS, F. (2000). Participación comunitaria y gestión alternativa de conflictos. Cuadernos de Trabajo Social (13), 231-252

DÁVILA, O. (2005). La gestión local de juventud en Chile. Politicas locales de juventud: experiencias en el Cono Sur. Recuperado el 31 de noviembre de 2012 de Friedrich Ebert Stiftung: http://library.fes.de/pdf-files/bueros/ chile/06788.pdf

DEPARTAMENTO DE ASESORÍA URBANA. (2006) Resumen ejecutivo: Estudio plan regulador comunal de Estación Central. Recuperado el 24 de noviembre de 2012, http://www.e-seia.cl/archivos/Resumen_Ejecutivo.pd

DIRECCION DE DESARROLLO COMUNITARIO-CENTRO DE ATENCION FAMILIAR. (DIDECO-CAF). (2008). Ariculando un diseño de una politica local de juventud. Santiago, Maipú

GOBIERNO DE CHILE. (n.d.). Ministerio de Planificación. Recuparado el 24 de octubre de 2012. Políticas
Ministeriales: http://www.gob.cl/cuenta-publica-2010/ ministerio-de-planificacion/politicas-ministeriales/

KETTNER, P. M., MORONEY, R. M., \& MARTIN, L. L. (1996). Designing and Managing Programs. Sage.

KRAUSKOPF, D. (2011). Ciudadanía joven y políticas: mirada restrospectiva y desafíos para el Estado de Chile. Seminario: Nuevas Ciudadanías, Democracia y Políticas Públicas (pp. 1-11). Santiago: Centro de Investigación Sociedad y Políticas Públicas (CISPO), Universidad de los Lagos.

LONG, N. (2001). Develpment Sociology: Actas Perspectives ( $1^{\text {st }}$ Edition ed.). New York: Routledge.

MANRíQUEZ, M., PALMA, P., \& TURINA, J. (2008). Diagnóstico juvenil comunal en temas de educación, empleo, participación sociocultural y politica, y políticas Departamento de Desarrollo Comunitario, Casa de la

MAZA, G. D. (1995). Construcción democrática, participación ciudadana y políticas públicas.

MINISTERIO DE DESARROLLO SOCIAL. (n.d.). Instituto Nacional de la Juventud. Recuparado el 24 de octubre, 2012. http://www.injuv.cl/portal/

MUNICIPALIDAD DE ESTACION CENTRAL. (2011) Minuta Consulta Joven. Santiago, Estación Central.

MUNICIPÀLIDAD DE ESTACIÓN CENTRAL. (2005). Plan de Desarrollo Comunal 2005-2010. Santiago.

PEREYRA, J. C. (n.d.). Carta del Presidente de FLACMA. Recuperado el 1 de diciembre de 2012. Federacion Latinoamericana de Ciudades Municipios y Asociaciones de Gobiernos Locales (FLACMA): http:// www.flacma.com/index.php?option=com_conten \&view=article\&id=902: carta-del-presidente-deflacma\&catid=35:informacion

PROGRAMA DE LAS NACIONES UNIDAS PARA EL DESARROLLO (PNUD). (2009). Informe sobre Desarrollo Humano Honduras 008/2009. De la Exclusión Social a la Ciudadanía Juvenil. Naciones Unidas, Honduras.

ROBERTS, B. (2001). Las nuevas políticas sociales en América Latina y el desarrollo de ciudadanía: Una perspectiva de interfaz. Agencia, conocimiento y poder.

RODRÍGUEZ, E. (2000). Juventud y políticas públicas en América Latina: experiencias y desafíos de la gestión institucional. Ultima Década (13), 25-58. 